\title{
Changes in Microbiota During Multiple Fermentation of Kefir in Different Sugar Solutions Revealed by High-Throughput Sequencing
}

\author{
Raúl Ricardo Gamba ${ }^{1} \cdot$ Takashi Koyanagi $^{1}$. Angela León Peláez ${ }^{2} \cdot$ Graciela De Antoni $^{2} \cdot$ Toshiki Enomoto $^{1}$ (D)
}

Received: 19 September 2020 / Accepted: 19 April 2021 / Published online: 7 May 2021

(c) The Author(s), under exclusive licence to Springer Science+Business Media, LLC, part of Springer Nature 2021

\begin{abstract}
Kefir is a fermented beverage produced through the activity of its grains, which is constituted by lactic acid and acetic acid bacteria and yeasts. We studied the bacterial succession during multiple fermentation of Argentinian kefir in brown sugar, purified molasses or high-test molasses, using $16 \mathrm{~S}$ high-throughput sequencing. Firmicutes was dominant (up to $98 \%$ of total population) in grains and beverages made from various sugar substrates, except in high-test molasses beverage, which was dominated by Proteobacteria (up to $78 \%$ of total population). Major bacterial species in Firmicutes were Liquorilactobacillus nagelii, Lentilactobacillus hilgardii/diolivorans and Lacticaseibacillus casei/paracasei, which are active in lactic acid fermentation. Proteobacteria comprised Acetobacter lovaniensis and Gluconobacter oxydans/roseus as major species, which are presumably responsible for the acetic acid formation in sugary kefir beverages. Bacteria differ in abundance depending on the sugar type, as revealed by the competitive dominances between L. nagelii and A. loveniensis. Purified molasses led to scarce acetic acid bacteria during fermentation, indicating that it is not a suitable substrate for their growth. Our results suggest that acetic acid (and/or ethanol) in sugary kefir modulates the succession and dominance of specific lactic acid bacteria. This study will provide valuable information for designing more sophisticated non-dairy fermented beverages with stable microbial properties.
\end{abstract}

\section{Introduction}

Kefir is a beverage made from fermentation of milk or sugar solutions with kefir grains. Kefir grains are a consortium of lactic and acetic acid bacteria and yeasts. Kefir beverage has multiple health benefits, including antitumor, antibacterial, antioxidant, and cholesterol-reducing properties [1-3].

There is stark contrast between our current knowledge of milk kefir and sugary kefir, in that the former is better known, better studied, and consumed more often [4]. Therefore, it is necessary to fully understand the microbial profile of sugary kefir to achieve a successful product with the best properties. Kefir has been studied for decades using various techniques. In the past, kefir and its microorganisms were studied using culture-dependent methods [5-7]. Classic

Toshiki Enomoto

enomoto@ishikawa-pu.ac.jp

1 Department of Food Science, Ishikawa Prefectural University, Nonoichi, Ishikawa 921-8836, Japan

2 Facultad de Ciencias Exactas, Cátedra de Microbiología, Universidad Nacional de La Plata, Calle 47 y 115, 1900 La Plata, Argentina microbiological studies suggest that bacteria constitute up to $90 \%$ of the microbial population in the grains [7]. Lactic acid bacteria (LAB) such as Lactococcus, Lactobacillus, Streptococcus, and Leuconostoc and acetic acid bacteria (AAB) such as Acetobacter have been described as major species $[5,6]$. Although these early studies provided substantial amount of information, their methods may be limited by low sequence coverage in the data. In recent years, cultureindependent methods have been increasingly used to provide more accurate and in-depth analysis while avoiding the limitations mentioned above. High-throughput DNA sequencing has led to a great advancement in research on microbial ecosystems such as those in fermented food [8, 9]. Indeed, this technique has been used to study the microbial population of kefir $[10,11]$. However, previous studies only focused on the microbial variety between grains with different origins, or between grains and their final products [10-13]. None of them investigated the bacterial succession during multiple cycles of fermentation. In our previous study, we conducted the culture-dependent analysis on bacteria inhabiting sugary kefir, and demonstrated that diverse bacterial succession might be occurring in three different sugar substrates (brown sugar, purified molasses, and high-test molasses) during 
seven cycles of repetitive inoculation [1]. We detected various LAB species including Liquorilactobacillus nagelii (formerly Lactobacillus nagelii), Lentilactobacillus hilgardii (formerly Lactobacillus hilgardii), Lentilactobacillus farraginis (formerly Lactobacillus farraginis), Liquorilactobacillus satsumensis (formerly Lactobacillus satsumensis), Schleiferilactobacillus harbinensis (formerly Lactobacillus harbinensis), Lentilactobacillus diolovorans (formerly Lactobacillus diolivorans) and Lacticaseibacillus casei/paracasei (formerly Lactobacillus casei/paracasei), and several AAB species including Acetobacter tropicalis, Acetobacter indonesiensis, Acetobacter lovaniensis, and Gluconobacter oxydans as major isolates.

In the present study, using the same three above-described sugar substrates, we analyzed the bacterial microbiota succession during multiple fermentation of sugary kefir using high-throughput $16 \mathrm{~S}$ ribosomal RNA gene (16S rDNA) sequencing. The microbial composition of kefir varied depending on the three-sugar medium, consistent with our previous result obtained through culture-dependent approach [1]. An interesting trade-off manner of the bacterial composition between specific lactic- and acetic acid bacteria species was observed depending on the sugar substrates, which was not found in our previous culture-dependent approach. Kefir beverage produced from sugar solutions could be beneficial for certain consumers such as vegans, lactose intolerant people, and people who are allergic to dairy products. In our previous study, we found that kefir fermentation improved functional properties of brown sugar-based (BS) kefir, purified molasses-based (PM) kefir and high-test molasses-based (HM) kefir [1]. The detailed bacterial microbiota characterized in this study will provide useful information for improving the quality and probiotic benefits of sugary kefir beverages.

\section{Materials and Methods}

\section{Samples and Inoculation of Kefir Grains}

Kefir grains and fermentation conditions used in this study were described previously [1]. Briefly, grains stored at $-80{ }^{\circ} \mathrm{C}$ were activated at $25^{\circ} \mathrm{C}$ for $24 \mathrm{~h}(10 \mathrm{~g}$ grains with $100 \mathrm{~mL}$ sugar solution). Three sugar solutions were used as growth media: $5 \%(\mathrm{w} / \mathrm{v})$ brown sugar (BS, Kanbayashi Co., Ltd., Osaka, Japan); 6.5\% (w/v) purified molasses (PM, Dai-Nippon Meiji Sugar Co., Ltd., Tokyo, Japan); and 6.5\% (w/v) high-test molasses (HM, Dai-Nippon Meiji Sugar Co., Ltd.). PM and HM are by-products from manufacturing of white sugar and raw sugar, respectively. These three solutions have a similar sugar concentration $\left(4.7-4.9^{\circ}\right.$ Brix $)$. The media were replaced by fresh solutions three to four times a week to maintain the microbial activity of the grains.
Activated grains were used for fermentation. The grains were incubated at $25{ }^{\circ} \mathrm{C}$ for $24 \mathrm{~h}$ in respective sugar solutions for seven consecutive fermentations (multiple cycles of fermentation). Each sample was named as sugar solution plus fermentation day (e.g. first day of fermented BS [BSD1] to seventh day of fermented BS [BSD7] and BS kefir grains [BSG]; PM beverages from PMD1 to PMD7 and grains PMG; HM beverages from HMD1 to HMD7 and grains HMG). After final fermentation, the grains were separated from beverage by filtration with a sieve of $1-\mathrm{mm}^{2}$ mesh size. Part of beverages at 1 st, 4 th and 7 th cycles of fermentations and their grains were used for DNA extraction.

\section{Metagenomic DNA Extraction}

Two-hundred milliliters of each sugar solution was inoculated with $20 \mathrm{~g}$ of kefir grains and incubated at $25^{\circ} \mathrm{C}$ for $24 \mathrm{~h}$, the most common time point at which kefir is prepared [14]. To extract DNA from the kefir, $0.5 \mathrm{~mL}$ of fermented products were added into tubes from the EZ-extract for DNA kit (AMR Co., Ltd., Tokyo, Japan) containing sterilized glass beads. Samples mixed with beads were shaken by vortex for $10 \mathrm{~min}$. Samples were incubated for $3 \mathrm{~min}$ at room temperature and shaken again for $10 \mathrm{~min}$. Aliquots of $175 \mu \mathrm{L}$ were transferred to new tubes. From here, DNA was extracted using NucleoSpin ${ }^{\circledR}$ tissue kit (Macherey-Nagel GmbH \& Co. KG, Düren, Germany) according to manufacturer's instructions. Briefly, $200 \mu \mathrm{L}$ of lysis buffer was added to samples and incubated at $70{ }^{\circ} \mathrm{C}$ for $10 \mathrm{~min}$. Next, $210 \mu \mathrm{L}$ absolute ethanol was added to each sample. Samples were poured into spin column and centrifuged. Columns were washed twice with wash buffer, and DNA was eluted with elution buffer pre-heated at $70{ }^{\circ} \mathrm{C}$. After elution, pure DNA samples were obtained. Kefir grains were disrupted in a mortar and resuspended in sterilized $0.8 \%(\mathrm{w} / \mathrm{v}) \mathrm{NaCl}$. Samples of $0.5 \mathrm{~mL}$ were used for DNA extraction as described above. The quality of extracted DNA was evaluated by $1.0 \%(\mathrm{w} / \mathrm{v})$ agarose electrophoresis.

\section{DNA Amplification and Sequencing}

Metagenomic DNA was used as template for PCR amplification with KOD FX DNA polymerase (Toyobo, Tokyo, Japan). The V3 and V4 variable regions of the bacterial $16 \mathrm{~S}$ rDNA were amplified by PCR. The primers for 1 st PCR amplification were designed according to Illumina library construction protocol, 341F (5'-TCGTCGGCAGCGTCA GATGTGTATAAGAGACAGCCTACGGGNGGCMGCAG -3', forward) and 806R (5'- GTCTCGTGGGCTCGGAGA TGTGTATAAGAGACAGGACTACHVGGGTATCTA ATCC-3', reverse). Each primer contains the specific adaptor sequence for Illumina MiSeq apparatus on $5^{\prime}$-ends, and the sequences annealing to $16 \mathrm{~S}$ rDNA were underlined. The 
conditions used for PCR amplification were $94{ }^{\circ} \mathrm{C}$ denaturation for $2 \mathrm{~min}, 35$ cycles of $98{ }^{\circ} \mathrm{C}$ for $10 \mathrm{~s}$ (denaturation), $50{ }^{\circ} \mathrm{C}$ for $30 \mathrm{~s}$ (annealing) and $68^{\circ} \mathrm{C}$ for $60 \mathrm{~s}$ (extension). All DNA was subject to a hot start at $94{ }^{\circ} \mathrm{C}$ prior to PCR amplification. Amplicons generated were purified using the Agencourt AMPure XP purification system (Beckman Coulter Genomics, Indianapolis, USA). Second PCR was performed using the primers in Nextera XT Index Kit v2 (Illumina ${ }^{\circledR}$, San Diego, USA). These primers contain the 8-bp multiplexing indexes, in addition to the Illumina MiSeq adaptor sequences, to facilitate the pooling and subsequent differentiation of samples. Purified PCR products were quantified using the Nanodrop 3300 Fluorospectrometer (Thermo Scientific, MA, USA). Equal amount of 16S rDNA V3-V4 amplicons from each sample were pooled and sequenced on an Illumina MiSeq (Illumina ${ }^{\circledR}$ ) according to Illumina protocols.

\section{S rDNA-Based Taxonomic Analysis}

Data obtained from the MiSeq analysis were processed and analyzed using QIIME pipeline software (version 1.8.0) [15]. The non-chimeric reads were clustered into operational taxonomic units at a $97 \%$ cutoff threshold. The taxonomic identification at the genus level was performed by the Ribosomal Database Project (RDP) classifier program (version 2.2) [16] equipped with the Greengenes $16 \mathrm{~S}$ rDNA database (version gg_13_8) [17]. In order to identify the closest species for representative sequences, we used the Basic Local Alignment Search Tool (BLAST) (version 2.2.20). Diversity (Shannon) and richness (Chao1) indices were calculated by sampling the obtained reads up to 53,580 sequences.

\section{Accession Number(s)}

The sequence data obtained from the MiSeq analyses were deposited under the DDBJ DRA database with the accession number DRA008253.

\section{Results and Discussion}

\section{Richness and Diversity Observed for Bacterial Microbiota of Sugary Kefirs}

In this study, a total of 631,104 reads were obtained, after chimeric reads and reads shorter than $250 \mathrm{bp}$ were filtered out. Rarefaction curves showed that enough sequencing reads have been obtained for downstream analysis (Fig. S1). Chao1 and Shannon indices were calculated to evaluate the bacterial richness and diversity, respectively, in sugary kefir and their grains (Fig. 1). Chao 1 richness was similar in BS (25.5, an average of 1st, 4th, 7th cycles of fermentation) and
PM kefirs (25.2), whereas it was higher in HM kefir (36.07) (Fig. 1a), and relatively higher value was observed from the early fermentation stage of HM kefir beverage (HMD1). Shannon index showed that the diversity of middle-fermentation stage of BS kefir beverage (BSD4) was relatively high (Fig. 1b). Species richness and diversity of grains and corresponding kefir beverages changed during the multiple cycles of fermentation. The richness and diversity indices of grains were either higher or lower than those of respective beverages. Marsh et al. [10] previously reported slightly different results, in that almost all four grains had higher richness and lower diversity than their respective sugary kefir.

\section{Succession of Acetic Acid Bacteria in Sugary Kefir}

Firmicutes, Proteobacteria, Actinobacteria and Bacteroidetes were detected in kefir beverages and grains, with Firmicutes (21-99\%) and Proteobacteria (0-78\%) being at relatively higher proportions (Fig. 2). Difference at the phylum level was observed for PM kefir beverages (PMD), since this sugar substrate does not maintain Proteobacteria during the multiple cycles of fermentation, despite similar Brix degree $\left(4.7-4.9^{\circ}\right)$ in all three sugar solutions. The majority of Proteobacteria comprised AAB (Acetobacter and Gluconobacter) as shown in Fig. 3. Therefore, the PM is a poor substrate for acetic acid fermentation. Contents of fat, nitrogen and carbohydrates in PM and HM are similar (data not shown). However, some minor essential components in PM might not be sufficient, compared to $\mathrm{HM}$, as nutritionally poorer white sugar is used as starting material for PM. By contrast, HM was evidently suitable for growth of Proteobacteria, which drastically increased from 22\% (HMD1) to $78 \%$ (HMD7) of total bacteria population during seven cycles of fermentations. Acetobacter lovaniensis constituted the majority of bacterial inhabitants (62\% for HMD4 and 75\% for HMD7, Fig. 3), indicating that this species contributes to majority of acetic acid production in this sugar substrate. This species was also dominant (approximately $10^{7} \mathrm{CFU} / \mathrm{mL}$ ) after seven cycles of fermentation of HM kefir in culturedependent analysis [1]. Brown sugar kefir could not sufficiently maintain A. lovaniensis, as this species decreased from 14\% (BSD1) to 1\% (BDS7) through multiple fermentations (Fig. 3). In summary, the high-throughput 16S rDNA sequencing analysis revealed that only HM supports the viability of $\mathrm{AAB}$, indicating that the sugary kefir beverages are microbially diverse depending on the sugar substrates, as shown in our previous culture-dependent study [1].

\section{Diversity of Lactic Acid Bacteria in Sugary Kefir}

Among other phyla of bacterial microbiota, such as Firmicutes, specific species of LAB were identified at stable proportions. Liquorilactobacillus nagelii was the major LAB 

(b) index of kefir grains (black spots) and resulting beverages (white spots) after consecutive fermentations in brown sugar (BS), purified molasses (PM), and high-test molasses (HM). Consecutive fermentation was carried out for 7 days and samand 7 th cycles of fermentation under the condition described in materials and methods. BSG, PMG and HMG indicate kefir grains of each substrate
Fig. 1 Chao1 (a) and Shannon ples were taken from 1 st, 4 th
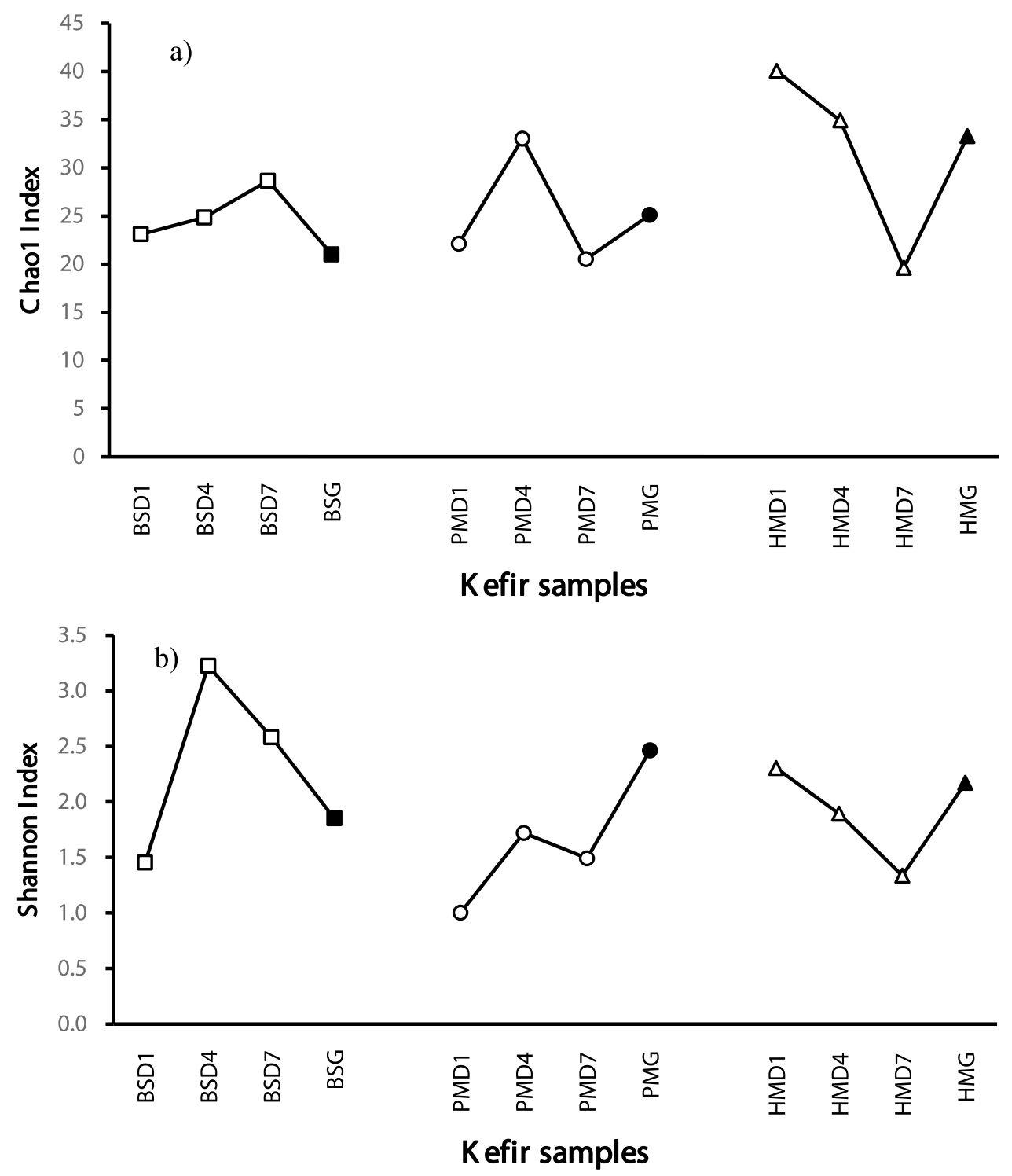

Fig. 2 16S phylogenetic composition of the bacteria of the sugary kefir and grains grown in brown sugar (BS), purified molasses (PM), and high-test molasses (HM) at phylum level. Consecutive fermentation was carried out for 7 days and samples were taken from 1st, 4th and 7 th cycles of fermentation under the condition described in materials and methods. BSG, PMG and HMG indicate kefir grains of each substrate

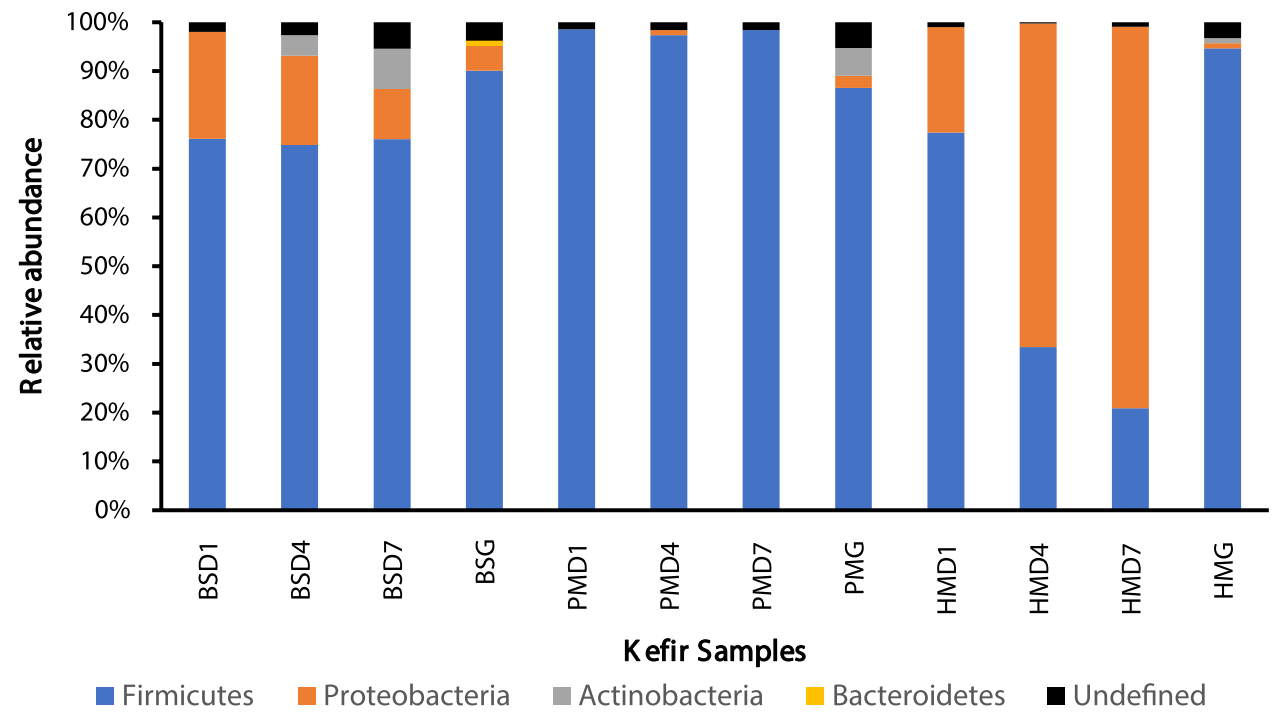


Fig. 3 16S phylogenetic composition of the bacteria of the sugary kefir and grains grown in brown sugar (BS), purified molasses (PM) and high-test molasses (HM) at genera/(species) level. The closest species dependent on the partially analyzed $16 \mathrm{~S}$ rDNA region was deduced using BLAST. Consecutive fermentation was carried out for 7 days and samples were taken from 1 st, 4 th and 7 th cycles of fermentation under the condition described in materials and methods. BSG, PMG and HMG indicate kefir grains of each substrate

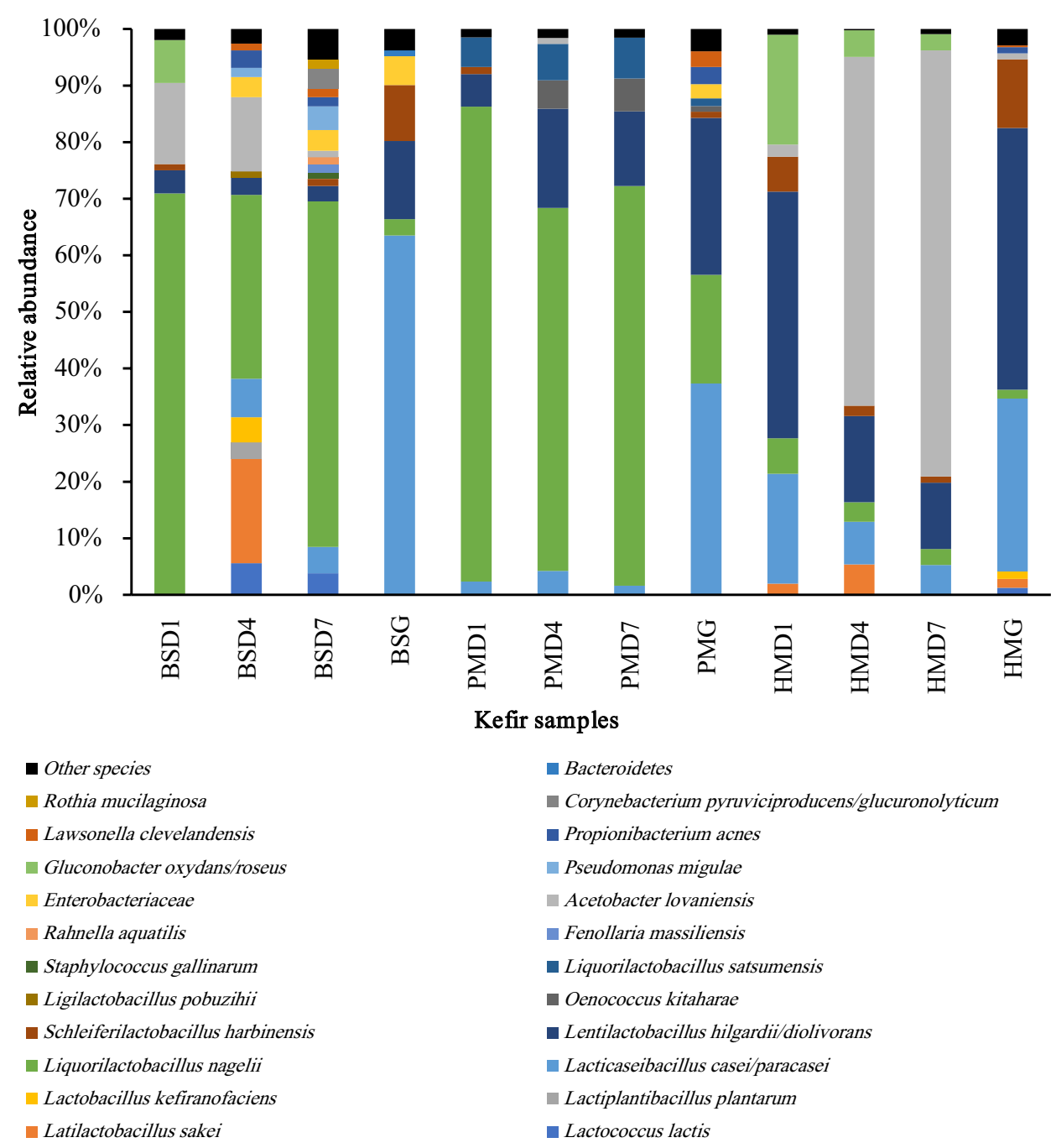

in BS kefir (33-71\% of total bacteria, BSD) and PM kefir (64-84\%, PMD) beverages. The result was comparable to that from our previous culture-dependent investigation [1]. A different pattern was observed for HM kefir beverages, which contained Lentilactobacillus hilgardii/diolivorans (12-44\%) and Lacticaseibacillus casei/paracasei (5-19\%) at higher abundance than L. nagelii (3-6\%). Although the major LAB species varied depending on sugar substrates, our result showed that $L$. nagelii, L. hilgardii/diolivorans and L. casei/paracasei played an important role in lactic acid formation in sugary kefir beverages. Previous studies reported that similar bacteria were detected in sugary kefirs from different countries [18], though different substrates were used such as brown sugar solution $(6 \mathrm{~g} / 100 \mathrm{~mL}$ [19] and $5 \mathrm{~g} / 100 \mathrm{~mL}$ [20, 21]), sucrose solution $(10 \mathrm{~g} / 100 \mathrm{~mL})$ with figs and a sliced lemon [22].

There was a trade-off manner of ratio between L. nagelii and $\mathrm{AAB}$ in microbiota of kefir from HM and other two sugar substrates. This might be explained by (i) acetic acid produced by AAB represses the viability of L. nagelii, or alternatively (ii) $L$. nagelii is more resistant than $L$. hilgardii/ diolivorans and L. casei/paracasei to ethanol, which is produced by yeasts and consumed by $\mathrm{AAB}$ coexisting in kefir. In either case, the growth of $\mathrm{AAB}$ would increase the acetic acid level and decrease the ethanol level, which might be disadvantageous for $L$. nagelii. We detected $10^{5}-10^{6} \mathrm{CFU} /$ $\mathrm{mL}$ of several yeast species (Saccharomyces cerevisiae and Pichia species) in culture-dependent analysis [1], indicating that the ethanol level in kefir beverage could affect growth of $\mathrm{LAB}$ with various ethanol tolerance. It is notable that Oenococcus kitaharae resided in PM kefir beverages (5-6\%), even though at a low percentage. Since the genus Oenococcus is a known ethanol-tolerant $\mathrm{LAB}$, it is expected to grow in sugary kefir in a trade-off manner with alcohol-degrading AAB. Indeed, O. kitaharae was quite scarce (no more than $1 \%$ ) in HM kefir beverage. We also isolated this species from PM kefir beverage in the previous culture-dependent analysis [1]. Another species Latilactobacillus sakei (formerly 
Lactobacillus sakei) was observed in BSD4, HMD1 and HMD4, but it was only detected occasionally with lower stability than the above three major LAB species (L. nagelii, L. hilgardii/diolivorans and L. casei/paracasei). Lentilactobacillus farraginis detected at high population (detected at $10^{5}-10^{7} \mathrm{CFU} / \mathrm{mL}$ in BS and PM kefir beverages) in the culture-dependent analysis was not observed as major constituents in the present study. This may be a selection bias due to either of the culture-dependent or PCR methodologies.

\section{Bacterial Microbiota in Different Sugary Kefir Grains}

Interestingly, all grains taken after seven cycles multiple fermentations comprised L. casei/paracasei (31-63\%) and $L$. hilgardii/diolivorans (14-46\%) at higher abundance than $L$. nagelii (2-19\%), showing a different trend between beverages and grains of sugary kefir. This difference was previously seen in milk kefir $[12,13]$. One notable exception was reported by Marsh et al. [10], who found high abundance of ethanol-producing bacteria Zymomonas in both grains and beverages of sugary kefir. Although different dominant bacteria have been reported in various studies, it is conceivable that the level of ethanol (produced by yeasts or Zymomonas) and/or acetic acid in kefir modulates the microbiota stability and later succession of specific LAB and other species mentioned above. Microbiota composition of sugary kefir could be affected by different sugar substrates as shown in this study, thus chemical characteristics in final beverage products could also be different. Future studies should include detailed analysis of chemical components and fungal microbiota including yeasts in these sugary kefirs.

Moreover, we found other bacterial groups with low abundance, as summarized in Table 1. Several species including Pseudomonas migulae (less than $4.21 \%$ of the population) were detected in kefir for the first time. Some of these minor species are considered opportunistic pathogens that affect

Table 1 Species detected in kefir for the first time

\begin{tabular}{|c|c|c|c|c|}
\hline \multirow[t]{2}{*}{ Phylum } & \multirow[t]{2}{*}{ Species } & \multicolumn{3}{|c|}{$\begin{array}{l}\text { Abundance (\% of total bacterial microbiota) in grains } \\
\text { and beverages }\end{array}$} \\
\hline & & $\mathrm{BS}$ & $\mathrm{PM}$ & $\mathrm{HM}$ \\
\hline Proteobacteria & Pseudomonas migulae & 4.21 & & \\
\hline Proteobacteria & Rahnella aquatilis & 1.28 & & \\
\hline Firmicutes & Ligilactobacillus pobuzihii & 1.07 & & \\
\hline Firmicutes & Staphylococcus gallinarum & 1.11 & & \\
\hline Firmicutes & Fenollaria massiliensis & 1.41 & & \\
\hline Actinobacteria & Lawsonella clevelandensis & & 2.78 & \\
\hline Actinobacteria & $\begin{array}{l}\text { Corynebacterium pyruviciproducens/glucurono- } \\
\text { lyticum }\end{array}$ & 3.48 & & \\
\hline Actinobacteria & Rothia mucilaginosa & 1.60 & & \\
\hline Phylum & Species & Lowe & & \\
\hline Firmicutes & Loigolactobacillus rennini & & & 0.04 \\
\hline Firmicutes & $\begin{array}{l}\text { Companilactobacillus farciminis/ heilongjiangen- } \\
\text { sis }\end{array}$ & & & 0.04 \\
\hline Firmicutes & Sporolactobacillus vineae & & 0.53 & \\
\hline Firmicutes & Streptococcus parauberis & & & 0.17 \\
\hline Firmicutes & Enterococcus raffinosus & & 0.20 & \\
\hline Firmicutes & Enterococcus faecalis & 0.55 & & \\
\hline Firmicutes & Kurthia gibsonii & & & 0.20 \\
\hline Firmicutes & Carnobacterium sp. & & & 0.03 \\
\hline Firmicutes & Anaerococcus tetradius/prevotii & 0.37 & & \\
\hline Firmicutes & Peptoniphilus sp. & 0.54 & & \\
\hline Firmicutes & Finegoldia magna & 0.56 & & \\
\hline Proteobacteria & Moraxella osloens & 0.78 & & \\
\hline Proteobacteria & Methylobacterium sp. & 0.75 & & \\
\hline Actinobacteria & Corynebacterium accolens & 0.84 & & \\
\hline Actinobacteria & Corynebacterium thomssenii & & & 0.03 \\
\hline Bacteroidetes & Prevotella melaninogenica & & 0.43 & \\
\hline
\end{tabular}

$B S$ brown sugar, $P M$ purified molasses, $H M$ high-test molasses. Highest percentages of mentioned bacterial species were indicated 
immunocompromised people, such as Rothia mucilaginosa, Fenollaria massiliensis, Staphylococcus gallinarum, Lawsonella clevelandensis, Rahnella aquatilis, and different Corynebacterium species [23-30]. The definite safety of kefir, however, has been established in the long history of this beverage. Therefore, a robust mechanism to suppress the activity of these harmful bacteria must exist intrinsically in kefir. Actually, we detected considerable antibacterial activity of the BS, PM, and HM kefir beverages on food-borne harmful bacteria Escherichia coli, Staphylococcus aureus, and Salmonella enterica subsp. enterica serovar Typhimurium in the previous report [1]. As mentioned above, a detailed analysis on chemical components and their relationship with microbiota will reveal more deepened mechanism of this antibacterial effect in the future studies. The great potential of sugary kefir as a good niche for probiotic LAB was also confirmed in this study. Contents of main sugars (glucose, fructose and sucrose) in substrates were enough consumed after fermentation (from 3.4 to $1.1 \%$ in BS kefir, from 2.5 to $0.099 \%$ in PM kefir and from 2.2 to $0.023 \%$ in HM kefir) (Table S1), therefore sugar contents should not be an obstacle to encourage the consumption of these kefirs. Further study is needed to apply the potential of above probiotic microbes to the food industry.

\section{Conclusions}

This report provides a detailed analysis on the successive fermentations of sugary kefir using high-throughput $16 \mathrm{~S}$ rDNA sequencing. We found that bacterial composition differs significantly between grains and beverages, and different type of sugars affect diversity of bacterial microbiota. One remarkable example is diversity in the succession of AAB. Three main LAB species, L. nagelii, L. hilgardii/diolivorans, and L. casei/paracasei, contribute to sugary kefir fermentation, although their abundance differs depending on the types of sugar substrate. We reasoned that the ethanol or acetic acid levels in fermentation medium and the tolerance of respective $\mathrm{LAB}$ species toward them influence the later succession of dominant species. It would be important to study the chemical components and fungal populations in sugary kefirs to obtain a thorough understanding of this millenium-old fermented product.

Supplementary Information The online version contains supplementary material available at https://doi.org/10.1007/s00284-021-02501-0.

Acknowledgements The authors thank to MEXT for scholarship of first author, Raúl Ricardo Gamba.

Authors Contributions RRG and TK performed the assays and data analysis. TE conceived of the study, and participated in its design and coordination and helped to draft the manuscript. ALP and GDA participated in the design of the study. All authors read and approved the final manuscript.

Funding None.

Data Availability All data generated or analyzed during this study are included in this manuscript.

\section{Declarations}

Conflict of interest The author declares that they have no conflict of interest.

Consent for Publication All authors have agreed to publish this manuscript.

\section{References}

1. Gamba RR, Yamamoto S, Sasaki T, Michihata T, Mahmoud A, Koyanagi T, Enomoto T (2019) Microbiological and functional characterization of kefir grown in different sugar solutions. Food Sci Technol Res 25:303-312. https://doi.org/10.3136/fstr.25.303

2. Rosa DD, Dias MMS, Grześkowiak $Ł M$, Reis SA, Conceição LL, Peluzio MCG (2017) Milk kefir: nutritional, microbiological and health benefits. Nutr Res Rev 30(01):82-96. https://doi.org/10. 1017/S0954422416000275

3. Zamberi NR, Abu N, Mohamed NE, Nordin N, Keong YS, Beh BK, Zakaria ZAB, Rahman NM, Alitheen NB (2016) The antimetastatic and antiangiogenesis effects of kefir water on murine breast cancer cells. Integr Cancer Ther 15(4):1-14. https://doi.org/ $10.1177 / 1534735416642862$

4. Market Research Future (2019) Kefir market share, sales, global production, consumption, top key players review and rapid growth by forecast to 2023. Web article. 2019. https://www.929thedrive. com/story/39792504/kefir-market-share-sales-global-productionconsumption-top-key-players-review-and-rapid-growth-by-forec ast-to-2023. Visited 14 May 2019.

5. Garrote GL, Abraham AG, De Antoni GL (2000) Inhibitory power of kefir: the role of organic acids. J Food Prot 63(3):364-369

6. Pidoux M, Marshall VM, Zanoni P, Brooker B (1990) Lactobacilli isolated from sugary kefir grains capable of polysaccharide production and minicell formation. J Appl Microbiol 69:311-320

7. Simova E, Beshkova D, Angelov A, Hristozova T, Frengova G, Spasov Z (2002) Lactic acid bacteria and yeasts in kefir grains and kefir made from them. J Ind Microbiol Biot 28(1):1-6. https://doi. org/10.1038/sj/jim/7000186

8. Koyanagi T, Nakagawa A, Kiyohara M, Matsui H, Yamamoto K, Barla $F$ et al (2013) Pyrosequencing Analysis of microbiota in Kaburazushi, a traditional medieval sushi in Japan. Biosci Biotech Biochem 77(10):2125-2130. https://doi.org/10.1271/bbb.130550

9. Quigley L, O'Sullivan O, Stanton C, Beresford TP, Ross RP, Fitzgerald GF, Cotter PD (2013) The complex microbiota of raw milk. FEMS Microbiol Rev 37:664-698

10. Marsh AJ, O'Sullivan O, Hill C, Ross RP, Cotter PD (2013) Sequence-based analysis of the microbial composition of water kefir from multiple sources. FEMS Microbiol Lett 348(1):79-85. https://doi.org/10.1111/1574-6968.12248

11. Walsh AM, Crispie F, Kilcawley K, O'Sullivan O, O'Sullivan MG, Claesson MJ, Cotter PD (2016) Microbial succession and flavor production in the fermented dairy beverage kefir. MSystems 1(5):e00052-e116. https://doi.org/10.1128/mSystems.00052-16 
12. Korsak N, Taminiau B, Leclercq M, Nezer C, Crevecoeur S, Ferauche C, Detry E, Delcenserie V, Daube G (2015) Evaluation of the microbiota of kefir samples using metagenetic analysis targeting the 16S and 26S ribosomal DNA fragments. J Dairy Sci 98:3684-3689. https://doi.org/10.3168/jds.2014-9065

13. Marsh AJ, O'Sullivan O, Hill C, Ross RP, Cotter PD (2013) Sequencing-based analysis of the bacterial and fungal composition of kefir grains and milks from multiple sources. PLoS ONE 8:693-671. https://doi.org/10.1371/journal.pone.0069371

14. Kök-Taş T, Seydim AC, Özer B, Guzel-Seydim ZB (2013) Effects of different fermentation parameters on quality characteristics of kefir. J Dairy Sci 96:780-789

15. Caporaso JG, Kuczynski J, Stombaugh J, Bittinger K, Bushman FD, Costello EK et al (2010) QIIME allows analysis of highthroughput community sequencing data. Nat Methods 7(5):335336. https://doi.org/10.1038/nmeth.f.303

16. Wang Q, Garrity GM, Tiedje JM, Cole JR (2007) Naive bayesian classifier for rapid assignment of rrna sequences into the new bacterial taxonomy. Appl Environ Microbiol 73(16):5261-5267. https://doi.org/10.1128/AEM.00062-07

17. De Santis TZ, Hugenholtz P, Larsen N, Rojas M, Brodie EL, Keller K, Andersen GL (2006) Greengenes, a chimera-checked 16S rRNA gene database and workbench compatible with ARB. Appl Environ Microbiol 72(7):5069-5072. https://doi.org/10.1128/ AEM.03006-05

18. Fiorda FA, de Melo Pereira GV, Thomaz-Soccol V, Rakshit SK, Pagnoncelli MGB, Vandenberghe LPS, Soccol CR (2017) Microbiological, biochemical, and functional aspects of sugary kefir fermentation - A review. Food Microbiol 66:86-95. https://doi. org/10.1016/j.fm.2017.04.004

19. Pidoux M (1989) The microbial flora of sugary kefir grain (the gingerbeer plant): biosynthesis of the grain from Lactobacillus hilgardii producing a polysaccharide gel. MIRCEN J Appl Microbiol Biotechnol 5(2):223-238. https://doi.org/10.1007/BF01741847

20. da Miguel MG, Cardoso PG, Magalhães KT, Schwan RF (2011) Profile of microbial communities present in tibico (sugary kefir) grains from different Brazilian States. World J Microbiol Biotechnol 27(8):1875-1884. https://doi.org/10.1007/s11274-010-0646-6

21. Magalhães KT, de Pereira GVM, Dias DR, Schwan RF (2010) Microbial communities and chemical changes during fermentation of sugary Brazilian kefir. World J Microbiol Biotechnol 26:1241-1250
22. Gulitz A, Stadie J, Wenning M, Ehrmann MA, Vogel RF (2011) The microbial diversity of water kefir. Int J Food Microbiol 151(3):284-288. https://doi.org/10.1016/j.ijfoodmicro.2011.09. 016

23. Bell ME, Bernard KA, Harrington SM, Patel NB, Tucker TA, Metcalfe MG, McQuiston JR (2016) Lawsonella clevelandensis gen. nov., sp. Nov., a new member of the suborder Corynebacterineae isolated from human abscesses. Int J Syst Evol Micr 66(8):2929-2935. https://doi.org/10.1099/ijsem.0.001122

24. Boiten KE, Jean-Pierre H, Veloo ACM (2018) Assessing the clinical relevance of Fenollaria massiliensis in human infections, using MALDI-TOF MS. Anaerobe 54:240-245. https://doi.org/ 10.1016/j.anaerobe.2018.03.008

25. Chudy-Onwugaje K, Vandermeer F, Quezada S (2019) Mimicking abdominal tuberculosis: abdominal abscess caused by Lawsonella clevelandensis in inflammatory bowel disease. Clin Gastroenterol Hepatol 17(8):e92. https://doi.org/10.1016/j.cgh.2018.06.017

26. Kuzdan C, Soysal A, Özdemir H, Coşkun Ş, Akman İ, Bilgen H, Özek E, Bakır M (2015) Rahnella aquatilis Sepsis in a Premature Newborn. Case Rep Pediatr. https://doi.org/10.1155/2015/860671

27. Maraki S, Papadakis IS (2015) Rothia mucilaginosa pneumonia: a literature review. Infect Dis 47(3):125-129. https://doi.org/10. 3109/00365548.2014.980843

28. Oliveira A, Oliveira LC, Aburjaile F, Benevides L, Tiwari S, Jamal SB, Silva A, Figueiredo HCP, Ghosh P, Portela RW, De Carvalho Azevedo VA, Wattam AR (2017) Insight of Genus Corynebacterium: Ascertaining the role of pathogenic and nonpathogenic species. Front Microbiol 8:1937

29. Tibra NK, Jalali S, Reddy AK, Narayanan R, Agarwal R (2010) Traumatic endophthalmitis caused by Staphylococcus gallinarum. J Med Microbiol 59(3):365-366. https://doi.org/10.1099/jmm.0. 011551-0

30. Williams AM, Collins MD (1990) Molecular taxonomic studies on Streptococcus uberis types I and II. description of Streptococcus parauberis sp. nov. J Appl Bacteriol 68(5):485-490. https://doi. org/10.1111/j.1365-2672.1990.tb02900.x

Publisher's Note Springer Nature remains neutral with regard to jurisdictional claims in published maps and institutional affiliations. 